\title{
Response of adenocarcinoma of the uterine cervix to chemoradiotherapy
}

\author{
ORIT KAIDAR-PERSON, SAWSAN YOSEFIA and ROXOLYANA ABDAH-BORTNYAK \\ Division of Oncology, Rambam Health Care Campus, Haifa 31096, Israel
}

Received July 20, 2014; Accepted February 19, 2015

DOI: $10.3892 / \mathrm{ol} .2015 .3105$

\begin{abstract}
The aim of the current retrospective study was to investigate the response of advanced cervical adenocarcinoma (AC) to definitive chemoradiotherapy. Uterine cervical cancer is one of the most common cancer types among females, with squamous cell carcinoma (SQCC) being the most prevalent histological type. The incidence of cervical $\mathrm{AC}$ and its variants has markedly increased in recent decades. The current understanding with regard to the treatment of cervical cancer has been established through studies in which the majority of the patients suffered from SQCC, while only a limited number of studies have focused on the treatment of AC. Therefore, the optimal treatment for uterine cervical AC remains unclear. In the present study, data were collected from the medical files of patients who were diagnosed with advanced uterine cervical AC and treated with chemoradiotherapy between 1998 and 2013. Data were also collected from a group of patients with SQCC for comparison with AC patients in terms of response and survival. A total of 68 uterine cervical cancer cases were included, including 29 AC patients and 39 SQCC patients. Compared with the SQCC subgroup, a higher number of AC patients required surgery following chemoradiotherapy due to a lack of response to the initial treatment (5\% vs. $31 \%$, respectively; $\mathrm{P}=0.0065)$. After a median follow-up period of 10 years, patients with AC exhibited shorter overall survival (7.4 years vs. 11 years for AC and SQCC groups, respectively; $\mathrm{P}=0.01)$. Differences in recurrence $(40.7 \%$ vs. $34.4 \% ; \mathrm{P}=0.79$ ) and disease-free interval (1.2 years vs. 2 years; $\mathrm{P}=0.11$ ) were not statistically significant. The results indicated that cervical $\mathrm{AC}$ is less responsive to chemoradiotherapy compared with SQCC.
\end{abstract}

\section{Introduction}

Cancer of the uterine cervix is the third most common gynecological cancer type and a cause of cancer-associated mortality

Correspondence to: Dr Orit Kaidar-Person, Division of Oncology, Rambam Health Care Campus, PO Box 9602, Haifa 31096, Israel

E-mail: o_person@rambam.health.gov.il

Key words: brachytherapy, carcinoma, chemoradiotherapy, high-dose rate, irradiation in females worldwide (1). The treatment of locally advanced uterine cervical cancer includes concomitant chemoradiotherapy and intracavitary brachytherapy as the definitive treatment. Cervical cancer consists of a several histopathological types, of which the most common type is squamous cell carcinoma (SQCC). The incidence of invasive uterine cervical adenocarcinoma (AC) and its variants has increased over the last few decades (1-3), and it has been demonstrated to be associated with poor prognosis. Possible explanations for the growing incidence are obesity and estrogen exposure (2). However, the majority of the current knowledge with regard to the treatment of cervical cancer has been established through studies in which the majority of patients suffered from SQCC. Only a limited number of studies have focused on the treatment of cervical AC; therefore, the present understanding regarding the optimal treatment of uterine cervical AC is limited. The aim of the present study was to investigate the response of advanced cervical AC to definitive chemoradiotherapy in comparison to that of cervical SQCC.

\section{Patients and methods}

Patients. A retrospective study was conducted using the medical records of consecutive patients with AC of the uterine cervix who had been treated at a single tertiary center (Rambam Health Care Campus, Haifa, Israel) between 1998 and 2013. No inclusion/exclusion criteria was used, and all patients were included. The patients had received concomitant external beam radiotherapy (EBRT) combined with weekly chemotherapy and intracavitary high-dose rate (HDR) brachytherapy. All the patients were treated with curative intent. In addition, the data of 39 patients with SQCC of the uterine cervix were also reviewed. As there were more patients with SQCC, the data of every third patient was collected, to avoid a bias. Medical records were reviewed to obtain information regarding disease stage, radiotherapy doses, response, time to recurrence and survival. Overall survival data were available for all the patients and were based on the results of the population census as recorded in the population registry of the Ministry of Interior of Israel. The study was approved by the ethics committee of Rambam Medical Center, Haifa, Israel.

Statistical analysis. The Kolmogorov-Smirnov test was used to test for normality of the distribution, and according to the results a parametric and non-parametric models were 
Table I. Clinical characteristics of patients.

\begin{tabular}{|c|c|c|c|}
\hline Characteristic & SQCC & $\mathrm{AC}$ & P-value \\
\hline Patients, $\mathrm{n}$ & 39 & 29 & \\
\hline Jewish decent, n (\%) & $35(89.7)$ & $21(72.4)$ & 0.11 \\
\hline $\begin{array}{l}\text { BMI, } \mathrm{n}(\%) \\
\leq 25 \mathrm{~kg} / \mathrm{m}^{2} \\
>25 \mathrm{~kg} / \mathrm{m}^{2}\end{array}$ & $\begin{array}{r}10(62.5) \\
6(37.5)\end{array}$ & $\begin{array}{r}6(30.0) \\
14(70.0)\end{array}$ & 0.09 \\
\hline $\mathrm{BMI}^{\mathrm{a}}$ & $26.05 \pm 6.3(23.5)$ & $30.31 \pm 6.1(32.0)$ & 0.05 \\
\hline Age at diagnosis, years ${ }^{a}$ & $54.4 \pm 13.36(52.5)$ & $54.34 \pm 13.07(54.0)$ & 0.98 \\
\hline \multicolumn{4}{|l|}{ Histological grade, n (\%) } \\
\hline 1 & $0(0.0)$ & $9(32.0)$ & 0.006 \\
\hline 2 & $13(43.0)$ & $11(39.0)$ & 0.79 \\
\hline 3 & $17(57.0)$ & $8(29.0)$ & 0.037 \\
\hline \multicolumn{4}{|l|}{ FIGO stage, n (\%) } \\
\hline I & $12(31.0)$ & $12(43.0)$ & 0.37 \\
\hline II & $24(62.0)$ & $11(39.0)$ & 0.09 \\
\hline III & $3(8.0)$ & $4(14.0)$ & 0.42 \\
\hline IV & $0(0.0)$ & $1(4.0)$ & 0.15 \\
\hline \multicolumn{4}{|l|}{ Treatment, $\mathrm{n}$} \\
\hline Brachytherapy & 0 & 1 & 0.15 \\
\hline Chemoradiotherapy (EBRT + brachytherapy) & 38 & 23 & 0.014 \\
\hline EBRT + brachytherapy only & 1 & 5 & 0.09 \\
\hline
\end{tabular}

${ }^{a}$ Data are presented as the mean \pm standard deviation. SQCC, squamous cell carcinoma; AC, adenocarcinoma; BMI, body mass index; FIGO, International Federation of Gynecology and Obstetrics; EBRT, external beam radiotherapy.

used. The differences between the two histology groups for continuous parameters (such as age) were evaluated by $t$-test or Mann-Whitney U test. Categorical parameters were evaluated by Fisher exact test or the Pearson $\chi^{2}$ test. Overall survival was defined as the time from the first day of treatment to mortality (all causes). Survivors were censored at the last follow-up. Time to tumor progression was defined as the time from the first day of treatment to the first recorded evidence of progression. Alive patients without progression were censored at last follow-up. Kaplan-Maier curves were used to illustrate main results. Two-tailed $\mathrm{P}<0.05$ was considered to indicate a statistically significant difference. Statistical analyses was performed using SPSS (Statistics Products Solutions Services) 21.0 software for Windows (IBM SPSS; Armonk, NY, USA).

\section{Results}

Patient characteristics. A total of 68 patients were included in the present study, including 29 patients with cervical AC and 39 patients with cervical SQCC. The median follow-up period was 10 years (range, $0.5-15$ years). The majority of patients were of Jewish descent $(89.7 \%$ in the SQCC group and $72.4 \%$ in the AC group). The disease stages and treatment of patients in the two groups are summarized in Table I. The rate of bulky disease $(>4 \mathrm{~cm})$ was similar between the groups.

Treatment strategies. For all the patients, EBRT was performed prior to HDR brachytherapy. Cisplatin $\left(40 \mathrm{mg} / \mathrm{m}^{2}\right)$ was used concomitantly with pelvic irradiation in all patients, with the exception of two patients in the SQCC group, for whom carboplatin was used (area under the curve, 2) due to renal insufficiency. In addition, concomitant chemotherapy was not administered to one patient in the SQCC group and 5 in the AC group due to comorbidities (Table I). Furthermore, three patients in each group did not receive the planned chemotherapy cycles due to acute gastrointestinal toxicity (diarrhea), which was the main indication to discontinue chemotherapy.

The intended treatment included external radiotherapy to the pelvis, at a dose of 45-50.4 Gy (25-28 fractions, 1.8 Gy per fraction, five fractions per week) using $6 \mathrm{MV}$ or $18 \mathrm{MV}$ beams, according to the protocol of the Division of Oncology, Rambam Health Care Campus. Depending on the patient's body habitus, two opposing (anterior-posterior) fields (for the majority of patients) or the 4-fields box technique (for those with a larger body habitus) were used. Patients with parametrium involvement were given a 5.4 Gy (1.8 Gy per fraction) boost with a central block in the vagina and uterine-cervical area. The average EBRT dose administered did not differ significantly between the groups (4914 cGY vs. $5055 \mathrm{cGY}$ for the AC and SQCC groups, respectively; $\mathrm{P}=0.19)$. HDR brachytherapy was performed using an $\operatorname{Ir}^{192}$ source. Prior to the first HDR brachytherapy fraction, the patients underwent intrauterine stent insertion and evaluation for response under anesthesia. The HDR fractionation scheme was planned for five fractions of 5-5.5 Gy each (depending on clinical 


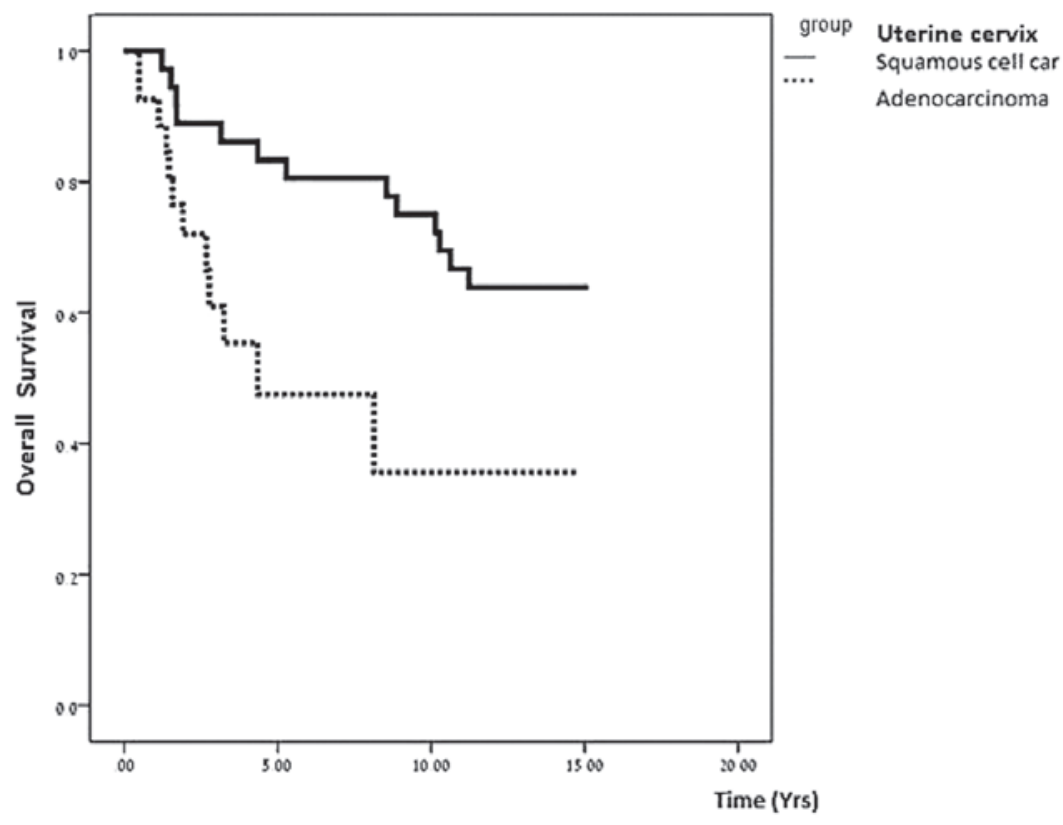

Figure 1. Survival curves for patients with adenocarcinoma and squamous cell carcinoma of the uterine cervix in the present study.

evaluation of tumor size). Treatment doses were prescribed at point A. HDR doses did not differ between the two groups.

In total, nine cervical AC patients required surgery following chemoradiotherapy due to residual disease, compared with two patients in the SQCC group (31\% vs. 5\%, respectively; $\mathrm{P}=0.0065$ ). No statistically significant difference in mortality rate was observed between the two subgroups (36\% vs. $44 \%$ for AC and SQCC groups, respectively; $\mathrm{P}=0.61$ ). The median follow-up period was 10 years, revealing that patients with cervical AC presented a reduced overall survival (7.4 years in the cervical AC group, vs. 11 years in the cervical SQCC group; $\mathrm{P}=0.01)$. Furthermore, the differences in recurrence rate $(40.7 \%$ vs. $34.4 \%$; $\mathrm{P}=0.79)$ and disease-free interval (1.2 years vs. 2 years; $\mathrm{P}=0.11$ ) were not statistically significant. However, significant differences were observed in overall survival between the two groups (Fig. 1). The majority of recurrences in the two groups were distant; four patients in the AC group exhibited local recurrence (within the irradiated field), and five patients exhibited distant recurrences (outside the irradiated field, including the lung, skeleton and liver), which was similar to that exhibited by patients of the SQCC group, where five patients exhibited local recurrence and six patients exhibited distant recurrences. Furthermore, a higher number of $\mathrm{AC}$ patients required surgical treatment for residual disease when compared with SQCC patients. These results indicate that cervical $\mathrm{AC}$ is less responsive to chemoradiotherapy than SQCC.

\section{Discussion}

The overall survival of patients with uterine cervical AC has been previously described to be poorer compared with that of SQCC patients, in studies conducted over the last two decades ago $(1,3)$. However, the management of these patients did not change, which may be attributed to the rarity of the disease and the lack of randomized trials. However, the incidence of invasive uterine cervical AC and its variants has increased over the last decades (2). Possible explanations for the growing incidence include obesity and estrogen exposure (4). In the present study, no statistically significant differences were observed between the AC and SQCC groups with regards to BMI (below or above $25 \mathrm{~kg} / \mathrm{m}^{2}$ ), with $70 \%$ of patients in the AC group having a BMI of $>25 \mathrm{~kg} / \mathrm{m}^{2}$, compared with $37.5 \%$ in the SQCC group.

The growing incidence and aggressiveness of $\mathrm{AC}$ of the uterine cervix, irrespective of the primary treatment $(2,4,6)$, requires the re-evaluation of treatment options for these patients. In the present study, more patients in the cervical AC group required surgery following chemoradiotherapy due to residual disease. The patients who were treated by chemoradiotherapy did not differ with regard to tumor size (bulky vs. non-bulky disease) or radiation dose between the SQCC and AC groups. Thus, the lack of response was likely to be due to the histological features of the tumor rather than the treatment. In contrast to the study by Eifel et al (2), which reported a trend towards improved survival in patients undergoing adjuvant hysterectomy (78\% vs. 71\%), no statistically significant differences in survival were observed in the present study $(\mathrm{P}=0.09)$. Additionally, surgery conferred no survival advantage in the present study. This may be due to the fact that surgery was performed as a treatment for unresponsive disease, rather than true adjuvant treatment.

Although the present study used a small sample size and was conducted retrospectively, its results support those of previous studies. Due to the growth in the prevalence of this disease, further investigation into treatment options is required (7); this may include the use of alternative chemotherapy regimens, such as the combined use of carboplatin and paclitaxel has been demonstrated to have radiosensitizing properties in various types of adenocarcinoma (8) and is a treatment strategy that is commonly used for uterine AC. 
Lee et al (8) evaluated this regimen in the context of pelvic radiotherapy in patients with SQCC of the uterine cervix. The study included 33 patients, who were treated with concurrent chemoradiotherapy, including two cycles of paclitaxel $\left(135 \mathrm{mg} / \mathrm{m}^{2}\right)$ and carboplatin (area under the time-concentration curve, $4.5 \mathrm{mg} \mathrm{x} \mathrm{min/ml)} \mathrm{at} \mathrm{four-week} \mathrm{intervals.} \mathrm{All} \mathrm{the} \mathrm{patients}$ received EBRT at a dose of 41.4-51.4 Gy (median, 50.4 Gy) to the entire pelvic area. Boost irradiation was administered to the cervix in 28 patients by brachytherapy at a dose of 25.6-43.3 Gy (median, $34.6 \mathrm{~Gy}$ ) and in 5 patients by external beam radiotherapy at a dose of 10.8-14.4 Gy (median, 14.4 Gy). The authors concluded that concurrent chemoradiotherapy with paclitaxel and carboplatin is effective to achieve pelvic control; however, the rate of toxicity was higher compared with that reported for platinum only (9). Acute hematological toxicity of grade 3 or 4 was reported in $\leq 61 \%$ of the patients (9). With current advances in EBRT treatment planning and the potential to perform intensity modulation planning (IMRT), it may be possible to reduce doses to the iliac crest in order to reduce hematologic toxicity (10). However, the use of IMRT in the treatment of intact uterine cervical cancer is not the standard of care. This may be due to the increased prevalence of the disease in developing countries and to pelvic organ movement and tumor regression, which increase the set-up variability when compared with other diseases, thus requiring larger margins and possible re-planning during the course of treatment. Therefore, data is available with regard to the use of IMRT as part of definitive chemoradiotherapy in uterine cervical cancer (11).

In conclusion, the current study shows that a higher number of $\mathrm{AC}$ patients required surgery following chemoradiotherapy due to a lack of response to the initial treatment and at a median follow up period of 10 years, patients with $\mathrm{AC}$ exhibited shorter overall survival. Based on the results of the present study, further multicenter prospective trials are required for the improvement of treatment in patients with uterine cervical AC. Advances in radiotherapy treatment planning and recent progress in chemotherapy regimens combined with radiotherapy in adenocarcinomas occurring at various sites may provide new opportunities for the investigation of novel effective treatments.

\section{Acknowledgements}

The authors would like to thank Mrs. Ronit Leiba (Rambam Health Care Campus) for the assistance in statistical analysis.

\section{References}

1. Fujiwara K, Monk B, Devouassoux-Shisheboran M. Adenocarcinoma of the uterine cervix: why is it different? Curr Oncol Rep 16: 416, 2014.

2. Eifel PJ, Burke TW, Morris M and Smith TL: Adenocarcinoma as an independent risk factor for disease recurrence in patients with stage IB cervical carcinoma. Gynecol Oncol 59: 38-44, 1995.

3. Smith HO, Tiffany MF, Qualls CR and Key CR: The rising incidence of adenocarcinoma relative to squamous cell carcinoma of the uterine cervix in the United States - a 24-year population-based study. Gynecol Oncol 78: 97-105, 2000.

4. Chen JL, Huang CY, Huang YS, et al: Differential clinical characteristics, treatment response and prognosis of locally advanced adenocarcinoma/adenosquamous carcinoma and squamous cell carcinoma of cervix treated with definitive radiotherapy. Acta Obstet Gynecol Scand 93: 661-668, 2014.

5. Lacey JV Jr, Swanson CA, Brinton LA, et al: Obesity as a potential risk factor for adenocarcinomas and squamous cell carcinomas of the uterine cervix. Cancer 98: 814-821, 2003.

6. Huang YT, Wang CC, Tsai CS, et al: Clinical behaviors and outcomes for adenocarcinoma or adenosquamous carcinoma of cervix treated by radical hysterectomy and adjuvant radiotherapy or chemoradiotherapy. Int J Radiat Oncol Biol Phys 84: 420-427, 2012.

7. Wakatsuki M, Kato S, Ohno T, et al; Working Group of the Gynecological Tumor: Clinical outcomes of carbon ion radiotherapy for locally advanced adenocarcinoma of the uterine cervix in phase $1 / 2$ clinical trial (protocol 9704). Cancer 120: 1663-1669, 2014

8. Lee MY, Wu HG, Kim K, Ha SW, Kim JS, Kim IA and Lee HP: Concurrent radiotherapy with paclitaxel/carboplatin chemotherapy as a definitive treatment for squamous cell carcinoma of the uterine cervix. Gynecol Oncol 104: 95-99, 2007.

9. van Hagen P, Hulshof MC, van Lanschot JJ, Steyerberg EW, van Berge Henegouwen MI, Wijnhoven BP, et al; CROSS Group: Preoperative chemoradiotherapy for esophageal or junctional cancer. N Engl J Med 366: 2074-2084, 2012.

10. Menkarios C, Azria D, Laliberté B, Moscardo CL, Gourgou S, Lemanski C, et al: Optimal organ-sparing intensity-modulated radiation therapy (IMRT) regimen for the treatment of locally advanced anal canal carcinoma: a comparison of conventional and IMRT plans. Radiat Oncol 2: 41, 2007.

11. Goldie SJ, Gaffikin L, Goldhaber-Fiebert JD, et al; Alliance for Cervical Cancer Prevention Cost Working Group: Cost-effectiveness of cervical-cancer screening in five developing countries. N Engl J Med 353: 2158-2168, 2005. 